\title{
Historia, investigación y discurso de la Psicología Positiva: Un abordaje crítico
}

\section{History, Research, and Discourse of Positive Psychology: A Critical Approach}

\author{
Luís Fernández-Ríos \\ Departamento de Psicología Clínica y Psicobiología, Facultad de Psicología, \\ Universidad de Santiago de Compostela, España \\ Manuel Vilariño Vázquez \\ Departamento de Psicología y Sociología, Facultad de Ciencias Sociales y del Trabajo, \\ Universidad de Zaragoza, España,
}

Recibido (18 de noviembre de 2014) Aceptado (30 de mayo de 2018)

\begin{abstract}
Resumen
La Psicología Positiva (PsP) ha alcanzado gran impacto académico, social y bibliográfico. No obstante, este éxito se ha visto empañado por el cuestionamiento de la originalidad de algunos de sus postulados, la cientificidad y relevancia social de los hallazgos de la investigación, a la vez que se han sugerido posibles riesgos derivados de sus intervenciones y aplicaciones. De este modo, el objetivo del presente trabajo es realizar una reflexión crítica y constructiva acerca de la situación actual de la PsP. Se concluye que la PsP debe ser más rigurosa históricamente mediante la adopción de una perspectiva de larga duración; llevar a cabo una construcción y diseminación del conocimiento fundamentada en la ética; desarrollar un discurso más realista dentro de una política de la mejora de la felicidad; y, por último, que los datos empíricos sobre los cuales se fundamenta sirven para justificar una ideología política neoliberal de la felicidad.

Palabras clave: psicología positiva crítica, cultura, felicidad, historia, larga duración.
\end{abstract}

\begin{abstract}
The Positive Psychology (PsP) has reached a great academic, social and bibliographic impact. Nonetheless, this success has been hindered by questioning the originality of some ideas; the scientific approach and the social relevance of the research findings. Likewise, possible risks derived from their interventions and implementations have been suggested. Thus, the objective of the present study is to conduct a critical and constructive reflection about the current situation of PsP. As a conclusion, the PsP must be more rigorous historically through the adoption of a long term perspective; carry out a construction and dissemination of knowledge based on ethic; develop a more realistic discourse within a policy of improving happiness and finally, empirical data that underpin the PsP are used to justify a neoliberal political ideology of happiness. Keywords: critical positive psychology, culture, happiness, history, long term.
\end{abstract}




\section{Introducción}

La Psicología Positiva (PsP, en adelante) ha logrado gran visibilidad social y éxito bibliográfico en los últimos años. Asimismo, ha forjado una profunda fascinación en múltiples profesionales, y una cierta decepción en otros muchos. Intenta proponer una perspectiva diferente acerca de la realidad psicológica del ser humano, en comparación con la denominada Psicología Negativa (PsN, de ahora en adelante) (Gillham \& Seligman, 1999; Seligman, 1998, 1999). En este sentido, la PsP representa el estudio científico de cómo las personas viven una vida digna, construyen fortalezas y virtudes para funcionar de una forma óptima y florecen psicológicamente en situaciones de vida cotidiana y de adversidad.

El ser humano parece hallarse en una perpetua duda acerca de si podrá ser tan plenamente feliz, como los psicólogos de la positividad le prometen. La PsP ilusiona a las personas con la posibilidad de alcanzar, con gran facilidad y sin especial esfuerzo personal, unas cuotas extremadamente altas de felicidad. Estas promesas de emociones positivas son, en demasiadas ocasiones, irreales e irracionales. Por esto, cuando no se consiguen, se corre el riesgo de caer en el escepticismo académico, el desencanto personal y la frustración social. La PsP, tal vez, se encuentre en los aledaños de la infracción del punto 3.04a del Ethical Principles of Psychologists and Code of Conduct (American Psychological Association [APA], 2017), que advierte a los psicólogos, incluidos, por supuesto, los positivos, que deben tomar "las medidas razonables para evitar dañar".

Por otro lado, a la PsP se le atribuye una pretendida originalidad e innovación en la creación de conocimiento sobre la felicidad. No obstante, una nada desdeñable cantidad de sus ideas ha sido tratada, con anterioridad, en obras clásicas de filosofía occidentales y orientales, o sencillamente por la sabiduría popular y el sentido común.

Este trabajo persigue el objetivo de realizar una aproximación crítica sobre algunas cuestiones, que juzgamos relevantes, de la PsP. Se trata de una línea de reflexión alineada con otra literatura redactada tanto en castellano (Fernández-Ríos, 2008; Fernández-Ríos \& Cornes, 2009; Fernández-Ríos \& Novo, 2012; Fernández-Ríos \& Vilariño, 2016; Pérez-Álvarez, 2013; Piña, 2014), como en inglés (Brown, Loma \& Eiroa-Orosa, 2017; McDonald \& Wearing, 2017). La estructura adoptada aquí, incluye cinco reflexiones críticas acerca de la PsP relacionadas con: la historia de la felicidad; la construcción del conocimiento de la mejora científica de las emociones positivas; la diseminación del conocimiento en las publicaciones de PsP; el discurso utilizado en la visibilización de la mejora científica de la felicidad; $y$, por último, la narrativa de la positividad en la política de ser feliz en una sociedad neoliberal.

\section{Crítica desde la Historia de la Felicidad}

La reflexión crítica que se desarrollará a continuación trasciende la falsa dicotomía entre presentismo e historicismo. Por eso, con el objetivo de centrar la cuestión, y superar un discurso profusamente repetitivo en la historia de las diferentes escuelas o movimientos, se parte de la perspectiva de algunos autores representativos de la Escuela de los Anales, École des Annales, francesa. Con ello se persigue ofrecer una aproximación innovadora para un nuevo enfoque del transcurrir temporal de la PsP. Concretamente, se hace referencia al término "larga duración" de Braudel (1958/2002). Este concepto se refiere aquí, a que la PsP está enraizada en el pasado de la historia del pensamiento filosófico, antropológico, político, literario, económico y religioso. En este sentido, resulta indispensable tener en cuenta el tiempo histórico de los conceptos relacionados con la PsP, pues forman parte de un pasado ejemplar, del cual se puede aprender mucho.

En contraposición al concepto de "larga duración" está el de "corta duración" (Braudel, 1958/2002), que aquí se refiere a que lo relevante para la PsP es lo publicado desde finales del siglo veinte en adelante. La corta duración incluye, aproximadamente, desde la aparición de los trabajos de Seligman (1998, 1999), y Gillham \& Seligman (1999). Lo que se suele incorporar en los libros de PsP representa una historia del presente, o perspectiva de corta duración. Todo lo anterior a 1998 sería PsN. Un ejemplo de esto lo hallamos en el reconocimiento de Snyder \& López (2007), acerca de que la PsP "representa una edad de oro potencial en la América del siglo XxI" (p. 6). Constituye, evidentemente, un error continuar defendiendo una historia de la PsP centrada en lo reciente, pues, como escribe Braudel (1958/2002), “el tiempo de corta duración es la más caprichosa y engañosa de las duraciones" (p. 151).

En consecuencia, se requiere una alternativa a lo tratado hasta ahora en la historia de la PsP. Gran parte de sus contenidos teóricos ya han sido pensados y escritos con anterioridad por alguien en algún lugar y en algún tiempo (Fernández-Ríos, 2008). De hecho, Seligman y Csikszentmihalyi (2000) reconocen, con gran acierto, que la PsP "no es una idea nueva" (p.13). Por tanto, dado que no representa algo original, la psicología de la positividad debe tener una historia de larga duración, que es la tesis defendida en el presente trabajo.

De acuerdo con esta perspectiva, el transcurrir histórico de la PsP se entremezcla tanto con la historia de las cosmovisiones, como con textos de antropología filosófica. Las reflexiones de Cicerón (2005) y las obras de Séneca (1996a, 1996b) son una buena muestra de ello. Otros ejemplos, de un marco histórico común de larga duración de la PsP, son el concepto de eudaimonía (Aristóteles, 1985, 
1994), y de hedonismo (Epicuro, 1996). Estos textos, entre otros muchos, deben ser leídos, pensados, interpretados y comprendidos, pues sólo hablan cuando se les interroga.

Conviene, asimismo, ser cuidadosos con los textos de corta duración acerca de la historia de la PsP, pues sus contenidos suelen ser borrosos, ambiguos y, en no pocas ocasiones, confusos y contradictorios. Sirva como ejemplo de razonamiento circular de Seligman (2008), admitir, por un lado, que aporta un nuevo concepto de salud, la "salud positiva" (p. 3); y, por otro, considerar que la PsP no es algo novedoso (Seligman \& Csikszentmihalyi, 2000). Alberga razón Kuhn (1962/2006, p. 249) cuando reconoce que los trabajos publicados de una disciplina suelen tergiversar su sentido histórico, en este caso, el de la PsP. Así, el texto de Snyder \& López (2007) considera que la historia de la PsP se halla en muchos autores clásico (p.e., Aristóteles, 1985, 1994; Epicuro, 1996), y poco más. Una evidencia fantasiosa de la historia de la PsP lo aporta el propio Seligman (2011), cuando reconoce que la PsP le llamó, "al igual que las zarzas ardientes llamaron a Moisés" (p. 96).

Existen otras múltiples muestras, de lo que se acaba de exponer. Una de ellas, que no aparece en las publicaciones, son las consolaciones, consolatio. Se trata de narrativas para compartir sentimientos de tristeza y, además, ayudar a superar duelos, y favorecer extraer beneficio de la adversidad (Lillo-Redonet, 2001). Ejemplos de textos de consolaciones son las de Plutarco (1986), Séneca (1996a, 1996b, 1996c), y la narración de Ovidio (2008) sobre su partida hacia un destierro forzado. Una muestra más del valor positivo de la historia reside en preguntarse por la contribuciones que el cristianismo puede hacer a la PsP (Johnson, 2017). Primero fueron las cosmovisiones religiones, y después la PsP, y no al revés. Las creencias religiosas, cuando se encuentran dentro de los límites saludables de la razón y el control emocional, hacen aportaciones positivas al proceso de vivir del ser humano. Tal es así, que las virtudes cardinales y teologales constituyen dimensiones del inventario de fortalezas psicológicas. De hecho, han sido incorporadas por los psicólogos de la positividad al Inventory of Strengths Values in Action (VIA) (Peterson \& Seligman, 2004).

Dado que la PsP incluye múltiples aspectos, de lo que han sido históricamente virtudes, ¿podría ser que se trate de una psicología de la virtud? Ciertamente, lo que hace la PsP a través del viA es aportar un vocabulario común, pero esto ya existía. Por ejemplo, se habla tanto de "fortalezas y virtudes" (Peterson \& Seligman, 2004, p. 6), como de "nueva ciencia del carácter" (p. 9). Realmente, la PsP podría ser poco más que una antropología filosófica de la virtud, cuyo contenido se encuentra, entre otros autores, en Platón (1986), Aristóteles (1985, 1994), Cicerón (2014) y Marco Aurelio (1983). Todas las cosmovisiones culturales ya han facilitado respuestas a la pregunta de qué hace que una persona sea buena y feliz. No en vano, Peterson \& Seligman (2004) aceptan que las fortalezas psicológicas habían sido enumeradas por "los filósofos durante siglos" (p. 13). En suma, históricamente, la PsP debería asumir un pasado amplio, y siempre dentro de una historia de larga duración.

\section{Crítica relacionada con la Construcción del Conocimiento de la Felicidad}

En sintonía con lo abordado en el anterior punto, no estaría de que más que la PsP también adoptase una perspectiva de larga duración para la historia de los conceptos o begriffsgeschichte (Koselleck, 2006/2012). Éstos no sólo poseen historia, además contienen y son guardianes de la historia de la felicidad. La PsP puede y debe reutilizar y reinterpretar el contenido relevante de los textos clásicos, adaptándolos al contexto actual. De hecho, es lo que han intentado llevar a cabo, en parte, Peterson y Seligman (2004).

Los hallazgos de múltiples investigaciones de la PsP resultan redundantes, así como concordantes con el sentido común. Por ejemplo, es obvio que lo positivo correlaciona con lo positivo, la felicidad con la positividad, el bienestar subjetivo con la esperanza, el optimismo con la felicidad, y la compasión con la espiritualidad. Pero, ¿existe algo realmente novedoso en estas conclusiones? Todo hace indicar que, en tales casos, la PsP no hace más que poner de manifiesto el sentido común cultural de la felicidad y de la esperanza de conseguirla. Evidencias de esto se hallan en las conclusiones acerca de que: existe una correlación positiva entre la gente feliz y la que florece (Lyubomirsky, King \& Diener, 2005); se pueden incrementar las emociones positivas prestando atención a las características positivas de la situación, y que seleccionar e implicarse en las emociones positivas incrementa la felicidad (Quoidbach, Mikolajczak \& Gross, 2015); se vincula el optimismo con una mejor salud emocional (Carver, Scheier, y Segerstrom, 2010); y, por último, la positividad disposicional predice la afectividad positiva (Caprara, Eisenberg \& Alessandri, 2017). No obstante, ha de reconocerse que no todo en PsP se relaciona con el sentido común en la investigación acerca de la positividad psicológica. Desde esta perspectiva, resulta adecuado que se centre en estudiar las experiencias subjetivas positivas, rasgos individuales positivos, e instituciones positivas.

Por otro lado, conviene formularse cuestiones como: ¿existe una patología de la emoción positiva en PsP? ¿Vale la pena, en contradicción con lo que postula la PsP, tener en cuenta tanto lo positivo, como lo negativo, en el transcurrir existencial cotidiano? Ambas cuestiones suponen una respuesta afirmativa. Sirvan como justificación, los siguientes ejemplos: a) una cierta dosis de pesimismo defensivo, incluso puede ser positivo y bueno para el proceso de vivir 
(Gruber \& Moskowitz, 2014; Kashdan \& Biswas-Diener, 2014; Lomas, 2016; Norem, 2001; Parrott, 2014); b) es posible tener demasiada felicidad, experimentar la felicidad en el momento equivocado, buscar la felicidad en los caminos equivocados, y experimentar tipos incorrectos de felicidad (Gruber, Mauss \& Tamir, 2011); c) el sistema afectivo positivo se puede llegar a asociar positivamente con algunas formas de psicopatología (por ejemplo, trastorno bipolar, uso de sustancias; Gruber et al, 2017); d) la promoción del afecto negativo puede ser útil para las personas. Pues, la tristeza o el humor negativo, que no es depresión, puede mejorar las relaciones interpersonales, incrementar la motivación para superar la situación, mejorar la precisión en los juicios de formación de impresiones y un estilo de pensamiento más detallado y atento y, además, mejora la memoria y el recuerdo de los detalles (Forgas, 2013, 2017); e) un énfasis excesivo en la necesidad de buscar emociones positivas, y evitar las negativas, puede acarrear implicaciones negativas, a través de rumiar sentimientos y experiencias negativas, para el bienestar psicológico de las personas (McGuirk, Kuppens, Kingston \& Bastian, 2017); y, por último, f) en pacientes con diversos cánceres de cuello y cabeza, no existían diferencias significativas en años de supervivencia entre los que se sentían abatidos y vencidos por la enfermedad, en comparación con lo que experimentaban pensamientos más positivos (Coyne et al., 2007).

Haciendo hincapié en este último apartado, se reconoce que existe una controversia en la teoría y práctica de la PsP con relación al cáncer. Por un lado está la perspectiva tradicional de la PsP, que sostiene que el optimismo es indispensable para la búsqueda de ayuda dirigida a superar dicha patología (Seligman, 2011). Por otro, están los críticos como Coyne \& Tennen (2010), quienes advierten de la existencia de una abundante literatura de carácter cuestionable sobre la relación de la PsP y el cáncer, pues la bibliografía disponible es inherentemente no científica. Por tanto, Coyne, Tennen \& Ranchor (2010) recomiendan ir a la pesquisa de evidencia empírica, si existe, en otro lugar, pero excluyendo la literatura de la PsP.

¿Podrían considerarse estos argumentos críticos acerca de la relación entre PsP y cáncer como una iatrogénesis en la puesta en práctica de la PsP? Se entiende aquí por iatrogénesis psicológica el daño subjetivo producido por un profesional de la psicología en el ejercicio de sus competencias profesionales durante el proceso de interacción con los pacientes. En este sentido, y con base en la crítica a la teoría y la práctica del pensamiento positivo llevada a cabo por Ehrenreich (2009/2011), podría sospecharse la posibilidad de una iatrogénesis clínica. Pues, las intervenciones desde la perspectiva del pensamiento positivo no parecen conseguir lo que prometen, por lo cual pueden operar como una trampa. De hecho, la ignorancia dentro de la teoría y práctica de la PsP parece ser altamente significativa. Por ejemplo, Park, Peterson \& Sun (2013) admiten que en las intervenciones de la PsP no se conoce, en la mayoría de los casos, ni cómo funcionan, ni cuáles son los mecanismos psicológicos a través de los cuales se provoca el cambio, "en comparación con los tratamientos tradicionales para los problemas clínicos" (p. 18).

Por lo anterior, se debe ser éticamente cuidadoso a la hora maximizar los beneficios y minimizar los daños en las evaluaciones e intervenciones (Barlow, 2010). En ocasiones, algunos psicólogos positivos, tal vez, podrían estar asumiendo compromisos y proponiéndose objetivos, que el código ético de la APA (2017) calificaría de "poco sensatos o imprudentes" (Principio C). Se corre, por tanto, el grave riesgo de que, aunque no siempre exista una evidente iatrogénesis clínica, sí tiene lugar una iatrogénesis sociocultural. Esta se materializa en que la PsP promete conseguir unas metas de felicidad a todos y cada uno de los individuos, que realmente no lo puede lograr. Es decir, en la sociedad neoliberal se promete, por un lado, la mejora científica de la felicidad y, por otro, se fabrican cada vez más nuevas patologías, lo que se denomina mongering (Frances, 2013/2014). Muestras patentes de esta perspectiva de la iatrogénesis sociocultural son los conceptos socialmente impuestos por la ideología de la PsP, como la "tiranía de lo positivo" (Held, 2002) o "el deber de ser feliz" (Bruckner, 2000).

\section{Crítica relacionada con la Diseminación del Conocimiento de la Felicidad}

La literatura científica es la "memoria institucional" de una disciplina e institución (APA, 2010). En el caso de la PsP, se trata de una memoria controvertida y contradictoria. La diseminación del conocimiento en libros y artículos científicos representa un proceso formal de dar a conocer resultados de investigaciones, siguiendo los estándares de construcción científica del conocimiento. En la actualidad, las revistas con factor de impacto se han convertido en el principal instrumento utilizado para tal fin. Sin embargo, los trabajos publicados en ellas redundan, una y otra vez, en las mismas teorías, que proporcionan resultados similares y uniformes. Es más, se van fusionado en el propio discurso de la PsP teorías que, en principio, poco tenían en común, como la de Bandura (2008) y la de Ryan \& Deci (2017).

El factor de impacto se emplea como la medida de la repercusión de una revista en la comunidad científica. Sirve para evaluar y comparar trabajos, autores, revistas e instituciones. Sin embargo, la política del factor de impacto ha sufrido duras críticas (Espeland \& Sauder, 2016; Santos- 
Rego \& Fernández-Ríos, 2016), e, incluso, se ha llegado a hablar de "publicación patológica" (Buela-Casal, 2014). Se incluyen bajo esta denominación, entre otras cuestiones, los diez mandamientos para incrementar las citas (BuelaCasal, 2002). Uno de ellos, representa lo que la APA (2010) designa como "publicación duplicada y publicación parcial de datos" (1.09), y "plagio y autoplagio" (1.10). No obstante, y a pesar de las duras críticas, el índice de impacto es considerado como algo académica valorado y políticamente correcto. En general, se trata, única y exclusivamente, de un estilo de pensamiento factor de impacto (Fernández-Ríos \& Rodríguez-Díaz, 2014), cuyo objetivo prioritario estriba en alcanzar la mayor cantidad posible de publicaciones con índice o factor de impacto, relegando a un segundo plano el auténtico valor científico y el impacto social de los hallazgos empíricos.

Atendiendo a esta idea, se revela la necesidad de adoptar un enfoque crítico de la PsP, que conlleva una constante vigilancia epistémica en el proceso de construcción del conocimiento para distinguir ciencia de pseudociencia. Con ello se pretende una PsP que mantenga una vigilancia reflexiva y crítica en el proceso de diseminación del conocimiento. En este sentido, no todo es positivo en el proceso de vivir, y lo negativo también puede resultar útil. Se trata, por tanto, de contemplar y promover el valor de lo negativo, de la adversidad, y de los sinsabores del proceso de vivir. No obstante, en la diseminación de conocimiento dentro de la PsP se evidencia un claro sesgo hacia las emociones positivas. Parece que todo lo relacionado con las emociones negativas está o debería ser prohibido, lo cual resulta, a todas luces, contraproducente. De todo lo negativo en el proceso de vivir se puede aprender para superar los contratiempos de la vida cotidiana. En el Preámbulo del Código Ético de la APA (2017) reconoce el deber de ayudar al público a desarrollar juicios y elecciones informadas. Sin embargo, no se puede sostener que la PsP actúe siempre así. Para alertar de esta realidad están los críticos de la psicología de la positividad. Así, para Held (2016) la división entre emociones e intervenciones positivas y negativas es algo "arbitrario, simplista e inexacto" (p. 34). En este mismo sentido, Widiger (2016) reconoce que la descripción y comprensión de los rasgos de personalidad como positivos o negativos es "demasiado simplista, y no se apoya en la evidencia empírica" (p. 263). Sin embargo, aunque Seligman \& Csikszentmihalyi (2000) reconocen que la PsP también se ocupa de la "reparación de las peores cosas de la vida" (p. 5), tiende a informar, casi exclusivasmente, acerca de los aspectos positivos del ser humano. Todo lo problemático o negativo es olvidado, despreciado y marginado dentro de epígrafe de PsN. Es como si la PsP abogase por una filosofía que preconiza un optimismo cruel, obsesivo e irracional, y que termina convergiendo en un ansia desbocada por cuotas inalcanzables de felicidad.

No todo en la teoría y práctica de la PsP es tan científico, cuantitativo y evidente, como en la diseminación del conocimiento dentro de la PsP, a veces, se quiere presentar. De este modo, se debe reconocer, como ya se ha señalado, por un lado, el poder positivo del pensamiento negativo; $y$, por otro, el lado oscuro de las emociones positivas. No se puede negar, ni obviar, la valía de los procesos emocionales, los pensamientos y las estrategias de afrontamiento negativas (Held, 2004, 2016). Ejemplos de esta realidad, sin ánimo de exhaustividad, se hallan en los trabajos de: Ford, Mauss \& Gruber (2015), en donde se concluye que la felicidad puede estar relacionada con desórdenes bipolares; Ford, Shallcross, Mauss, Floerke \& Gruber (2014), que aceptan que sobrevalorar la felicidad en poblaciones clínicas se relaciona con mayor sintomatología depresiva; Ford \& Mauss (2014), quienes aportan evidencia acerca que querer sentirse cada vez más feliz puede llevar a resultados negativos, incluyendo menor bienestar, peor salud psicológica y, paradójicamente, menos felicidad; y, por último, Liu et al. (2016), que reconocen que la felicidad y la infelicidad no parecen tener ningún efecto directo sobre la mortalidad de las mujeres.

En suma, la diseminación del conocimiento de la felicidad, si pretende ser científica y socialmente útil, no debería ceñirse a los aspectos considerados como positivos del ser humano, debido a que, quizás, lo erróneamente denominado negativo podría, en realidad, resultar productivo para la felicidad del ser humano.

\section{Críticas desde la Psicología del Discurso}

Tan importante para la PsP como su evidencia empírica, es su visibilidad social, académica y científica. El discurso de la PsP consiste en cualquier clase de interacción lingüística, hablada o escrita, interpersonal o por medio de las redes sociales, a través de la cual, formal o informalmente, se visibiliza y publicita el contenido teórico y práctico de la mejora científica de la felicidad. Por tanto, el lenguaje de la ciencia de la felicidad incluye significados compartidos, políticamente utilizados.

En las narrativas de la PsP abundan los conceptos denominados viajeros (Bal, 2002/2009), o nómadas, como por ejemplo: felicidad, emoción positiva, significado de la vida, bienestar psicológico, y bienestar emocional. En unos casos, resulta altamente complejo, cuando no imposible, diferenciarlos entre sí; $y$, en otros, se pueden incluso utilizar, y se utilizan, como sinónimos. Además, forman parte de discursos políticos, psicológicos, educativos, sociológicos, ecológicos, filosóficos, religiosos, y económicos. Discurso 
que suelen estar llenos de un imperialismo ideológico, en nombre de la ciencia de la felicidad universal. En esta línea, por imperialismo ideológico de la felicidad se entiende la imposición de una forma de conceptuar, categorizar y evaluar la felicidad en las culturas de las sociedades neoliberales occidentales, a todas las demás culturas. Parece tratarse de una ideología de la felicidad en las sociedades western, educated, industrialized, rich, y democratic (WEIRD; Henrich, Heine \& Norenzayan, 2010), que se supone válida y útil para toda cultura, tiempo y lugar.

Por tanto, el lenguaje de la PsP conlleva una gran carga ideológica y cultural, esto es, no es neutro, y, además, carece de significados únicos. Un ejemplo de esto es el juego discursivo que se hace con los conceptos mindfulness, felicidad, emoción positiva, significado de la vida, sentido del proceso de vivir, bienestar subjetivo, vida plena, etc. Esto provoca grandes problemas en la investigación. Una muestra de este caos terminológico se halla en el metaanálisis de Bolier et al., (2013) acerca de la efectividad de las intervención en PsP. Incluye conceptos tales como intervenciones de autoayuda, entrenamiento en grupo y terapia individual, pero se excluye de la búsqueda bibliográfica, entre otros, conceptos ejercicio físico, revisión de vida, mindfulness, meditación y perdón. Por esto, es tan difícil, por no decir imposible, comparar diferentes estudios de revisión en PsP, pues el concepto de mindfulness, en demasiadas ocasiones, se entremezcla con abundante contenido de la PsP (Ivtzan \& Lomas, 2016). Así pues, en unos meta-análisis se incluye el concepto de mindfulnees (Sin \& Lyubomirsky, 2009), mientras en otros es excluido (Bolier et al., 2013). Esto genera demasiada confusión en la integración del conocimiento de la psicología de la positividad. El trabajo de Diener et al. (2017) refleja con claridad este desconcierto teórico y empírico, en él se citan los dos meta-análisis anteriormente referidos para defender la utilidad unitaria de las intervenciones en PsP y bienestar subjetivo. Que uno incluya el concepto de mindfulness, y otro no, parece ser, en este caso, algo secundario. Sin embargo, realmente se trata de algo muy relevante e importante.

Curiosamente, tampoco la PsP tiene claro lo que significa positivo. Como prueba de esta confusión, Sheldon (2011) menciona cuatro significados de positivo en la PsP, a saber: hacer ciencia de lo positivo del ser humano; suponer que la naturaleza humana es inherentemente buena, es decir, positiva; centrarse en aspectos apreciables y admirables del ser humano, por tanto, positivos; $\mathrm{y}$, por último, estudiar el extremo positivo de tópicos particulares (por ejemplo, el perdón, en vez de la venganza). Esta confusión se agranda a la luz de las conclusiones de Pawelski (2016a, 2016b), cuando señala que el concepto de positivo puede contener, por lo menos, seis acepciones dispares dentro de la PsP; o que diferentes psicólogos positivos delimitan el dominio de lo positivo en formas distintas.

Por otro lado, en el discurso de la positividad se detectan demasiados ejemplos de lo que Haslam (2016) denomina como conceptos creep. Se trata de términos deslizados, arrastrados o deformados para alcanzar un significado extendido o ampliado y, consecuentemente, adaptables a múltiples contextos ideológicos, religiosos, teóricos, y culturales. Dichos conceptos se pueden utilizar para definirlos abarcando más síntomas o gravedad de la normal, aplicándolos a nuevos campos de la vida cotidiana. Por ejemplo, en PsP se puede considerar un episodio de tristeza cotidiana pasajera, como un gran problema a tratar. Aquí se produce un proceso a través del cual el significado de tristeza se establece con poca rigurosidad, ampliando su extensión para abarcar mayor sintomatología de la cotidiana y normal. Mientras que esta tristeza para la PsN representa un problema pasajero y normal, para la PsP consiste en una falta de realización del potencial de desarrollo óptimo del ser humano. Una consecuencia extrema de este proceder, sería estar triste por no ser todo lo feliz, que dicen los psicólogos de la positividad que debiera ser. Dos manifestaciones de esta confusión conceptual son las siguientes. Por un lado, no se puede establecer una diferencia diáfana entre emoción normal y patológica, por ejemplo, tristeza y depresión (Scherer \& Mehu, 2015); y, por otro, está la controversia de si se pueden experimentar emociones mezcladas o mixtas de forma simultánea o no (v. gr., estar triste, y estar alegre) (Larsen, 2017). Como muestra de emociones reales mezcladas, se escribe en el Diagnostic and statistical manual of mental disorders. 5th ed. (DSM-v; American Psychiatric Association, 2014), haciendo referencia al trastorno bipolar I: "El dolor del duelo puede ir acompañado de humor y emociones positivas que no son característicos de la intensa infelicidad y miseria que caracterizan a un episodio de depresión mayor" ( $\mathrm{p}$. 126). Otros ejemplos de estos conceptos psicológicos en expansión son abuso, trauma, trastorno mental, adicción, y prejuicio. En el caso del concepto de trastorno mental se extiende hasta incluir una serie de personas, que hace, por ejemplo, veinte años, se hubiesen considerado normales. Esto se relaciona con el concepto de disease mongering; es decir, fabricación de la categorización de nuevos sufrimientos o mercantilización de las enfermedades, en este trabajo, relacionadas con las emociones negativas y los déficits de felicidad (Frances, 2013/2014). Así, una persona que pasa por unos días tristes como consecuencia de la muerte de un ser querido, parece que ya sufre una patología psíquica, entiéndase alguna clase de trastorno mental, y que requiere de diagnóstico e intervención. Con la cual, el concepto de trastorno mental se expande e incluye cada vez más sufrimientos, más vulnerabilidades, $y$, por 
tanto, nuevas categorías de personas enfermas con una psicopatología de tristeza o de ansiedad. En realidad, se transforman o deforman los conceptos de tristeza y ansiedad normales, para convertirlos en una epidemia de problemas de supuestos trastornos mentales diagnosticables y tratables (Horwitz \& Wakefield, 2007). Esto implica que se amplía el número de personas sobre las cuales se pueden llevar a cabo intervenciones positivas. Similares razonamientos se pueden hacer con los otros términos de abuso, trauma, adicción, y prejuicio.

En conclusión, existen en el discurso de la PsP demasiados juegos del lenguaje, en el sentido de Wittgenstein (1953/2017). Estos juegos se plasman en el modo en que la PsP utiliza las palabras felicidad, satisfacción vital, eudaimonía, hedonismo, salud positiva, salud mental positiva. Hay algo en común entre todos ellos, aunque de una forma difusa y borrosa. Su significado está en cómo se conceptúan en cada caso concreto en el discurso de la positividad. Parece que, en demasiadas ocasiones, en las narrativas de la PsP, "el lenguaje bromea" (Wittgenstein, 1953/2017, parte I, y38); es decir, el discurso lingüístico se mueve en un cierto vacío, carece de una clara utilidad práctica y se pierde en narrativas confusas.

\section{Críticas desde la Perspectiva de una Política de la Felicidad}

Seligman (2011) reconoce que "siempre hay política detrás de la psicología positiva" (p. 259). Asimismo, más allá del discurso de la PsP, se verifica una realidad social en la que las personas sufren por las injusticias sociales, las desigualdades de clase, los abusos de poder y la corrupción. La investigación acerca de la felicidad debería ser útil para establecer políticas que la fomenten. No obstante, ¿puede aportar la PsP alguna solución para estos problemas? Es más, ¿podrían resultar perniciosas o incluso patológicas las políticas de la felicidad?

En este sentido, y sin ánimo de exhaustividad, se pueden señalar cuatro cuestiones acerca de la aplicación política de la PsP o de la política de la felicidad. En primer lugar, la acción política para la mejora científica de la felicidad puede resultar patológica cuando consiste en un paternalismo político, que conlleva la intromisión injustificada en la vida de las personas. El paternalismo político de la PsP consistiría en fomentar un estilo de liderazgo y hacer política, que, infantilizando a la ciudadanía, orienta la toma de decisiones acerca de qué deben y tienen qué hacer, y cómo realizarlo para ser necesariamente felices.

Un segundo aspecto radica en que la política de la felicidad contribuye a generar una cultura neoliberal de creación de la necesidad de ser cada vez más feliz. El neoliberalismo en la PsP es, ante todo, una ideología compuesta por unas prácticas político-económicas de la mejora científica de la felicidad dentro de un marco institucional de propiedad privada, y libre mercado. Admite que la mejor manera de promover el bienestar del ser humano es fomentando el libre desarrollo de las capacidades y de las libertades individuales. De alguna forma, el fin de la optimización del desarrollo personal justifica los medios. El neoliberalismo político y psicológico provoca una autorrealización impuesta, como una nueva forma de opresión. De hecho, se considera que la mejora científica de las emociones positivas genera un neocapitalismo de la felicidad. Aquí es, siguiendo a Adorno (1970/1980), donde interviene la industria de la cultura de las emociones positivas, "planificando la necesidad de felicidad y explotándola" (p. 405). En este contexto, podrían surgir algunos conflictos. Así, la APA (2010) reconoce que los "intereses económicos y comerciales" (1.12) pueden empañar la objetividad del conocimiento psicológico. En suma, la mejora científica de la felicidad en la sociedad neoliberal establece su propia verdad individual, social, teórica y práctica para el gobierno de las emociones positivas. Una muestra de esta filosofía de la positividad es el síndrome del bienestar (Cederström \& Spicer, 2015), referido a que cuanto más se esfuerzan los individuos en su contexto cultural por maximizar su bienestar y felicidad, más alienados y frustrados se sienten.

El tercer aspecto a tener en cuenta hace referencia a que se pueden cometer graves errores en las políticas de promoción de la felicidad. El altruismo y la empatía-compasión forman parte de las fortalezas psicológicas del Inventory of Strengths Values in Action (VIA; Peterson \& Seligman, 2004). No obstante, ¿podría el altruismo derivar en algo patológico y con consecuencias perversas? Todo hace indicar que sí. En esta línea, Oakley (2013) habla de altruismo patológico, que se puede conceptuar como "una conducta en la que los intentos de promover el bienestar de otro, $u$ otros, resultan, en cambio, en un daño que un observador externo concluiría que era razonablemente previsible" ( $\mathrm{p}$. 10408). El propio Oakley (2013) lo ejemplifica mediante la situación que se relata a continuación. Una persona X se vuelve adicta a los analgésicos. Cuando sufre abstinencia, otro individuo $\mathrm{Y}$, altruistamente, consigue más analgésicos para ayudar a que se sienta mejor, y la excusa o encubre ante un supervisor o un técnico de salud competente. $Y$ quiere ayudar a la persona $X$, pero realmente está ocultando, protegiendo o estimulando su enfermiza adicción. En este caso, un altruismo bien intencionado de $\mathrm{Y}$, se vuelve patológico para $\mathrm{X}$.

También se puede hablar de empatía patológica y perversa (Ferguson, 2016). Un ejemplo, del lado oscuro de la empatía se suele producir en personas que manifiestan una alta actitud empática, como recurso para explotar a otras personas (Wood, Emmons, Algoe, Froh, Lambert, y 
Watkins, 2016). Otra manifestación patológica de la empatía (Klimecki \& Singer, 2012) se produce cuando se ayuda una y otra vez a las mismas personas, pero ellas no hacen nada, pudiendo realizarlo, para superar su situación. Esto suele generar una fatiga de la compasión o fatiga de aflicción empática, que implica la consecuencia perversa de que cierta ciudadanía abandone conductas prosociales.

El cuarto, y última aspecto, de un carácter más positivo que los anteriores, es el prometedor concepto de riqueza psicológica de las naciones (Oishi, 2012). Este concepto hace referencia al capital psicológico colectivo del mundo interno de los ciudadanos, esto es, la riqueza psicológica de una sociedad reside en la suma de la riqueza psicológica de sus individuos. Se trata, por tanto, de extrapolar la idea de individuo sano y feliz a la sociedad saludable y con un elevado índice nacional de felicidad bruta. En relación con esta idea, se halla el conocido ejemplo de la puesta en práctica de una política para la supuesta mejora de la felicidad en el Reino de Bután. Por ciento, la política de la felicidad nacional bruta defendida en el Reino de Bután, ya se interconecta en este la teoría y práctica de la PsP. El trabajo de Adler \& Seligman (2016) es un buen ejemplo de ello.

En conclusión, una política de la mejora científica de la felicidad puede acarrear graves problemas. Estos van de un paternalismo intrusivo y una política neoliberal del negocio de la felicidad, hasta los aspectos negativos de la promoción obsesiva del altruismo o la empatía. Sin embargo, se atisba una esperanza teórica a través del discurso de la riqueza psicológica de las naciones, $\mathrm{y}$ todo lo que ello puede implicar para el diseño de políticas de bienestar. Con todo, ya se han llevado a cabo a la largo de la historia numerosos esfuerzos para construir sociedades sanas con ciudadanos felices. Tal y como reconoce Bok (2010), en los indicadores actuales del diseño de políticas públicas para la mejora de la felicidad, "simplemente se hacen eco de lo que algunos filósofos o teólogos han dicho hace siglos" (p. 205).

\section{Conclusiones}

A continuación se refieren las conclusiones que se pueden extraer del presente trabajo. En primer lugar, la PsP posee una historia de larga duración todavía por escribir. Esta orientación se fundamenta en la evidencia empírica que emana de la interpretación de los denominados textos clásicos, tanto occidentales como orientales. Sería deseable, en consecuencia, el desarrollo de una historia interdisciplinar de los esfuerzos llevados a cabo por el ser humano para la mejora de la felicidad. Segunda, la PsP en su proceso de construcción del conocimiento psicológico debe contemplar las emociones negativas y, por tanto, contar con la PsN.
No se puede separar lo positivo y lo negativo en el proceso cotidiano de vivir. Tal vez fuese del todo indeseable una vida sin emociones negativas. Realmente, éstas resultan útiles para llevar una vida con significado. Sencillamente se trata de aprender de la adversidad. En tercer lugar, se debe tener en cuenta el riesgo que conlleva la política del factor de impacto y la publicación patológica para la diseminación de resultados carentes de relevancia científica e impacto social. Cuando los investigadores se centran en conseguir publicaciones con factor de impacto, la cantidad de trabajos, sustituye a la calidad y creatividad. De este modo, si la ética en la investigación se estima imprescindible, no lo es menos la necesidad de establecer una vigilancia reflexiva y crítica en el proceso de diseminación del conocimiento. Parece que existen demasiados resultados positivos, mientras escasea la crítica reflexiva acerca de los mismos. En cuarto lugar, se halla el tema del discurso de la PsP, tan fácilmente manipulable para muy diferentes intereses y fines. Se debe asumir, por tanto, el imperativo académico, científico, y social de utilizar un discurso narrativo de la PsP que sea teóricamente equilibrado, argumentativamente ético, ideológicamente prudente, socialmente responsable, y filosóficamente reflexivo. Por último, y quinto lugar, se debe reconocer la necesidad de ser más cuidadosos, que en la actualidad, con ciertas implicaciones ideológicas y políticas de la mejora científica de la felicidad, y no transformar a la PsP en una industria cultural de la política neoliberal. Si la industria cultural de la felicidad predomina sobre el conocimiento empíricamente fundamentado, la PsP corre el riesgo de desorientarse académicamente y de ser políticamente manipulada.

A pesar de las críticas, la PsP goza de buena salud, al menos, en lo concerniente a su impacto académico y social, y éxito bibliográfico. No cabe duda, el discurso, la investigación y las políticas de la PsP continuarán creciendo en el futuro inmediato. A su alrededor pululan copiosos intereses creados a escala intelectual, académica, y de mercado de la felicidad. Sin embargo, adolece de múltiples elementos y aspectos lo suficientemente cuestionables como para despertar en muchos cierta, cuando no profunda, decepción. De este modo, aunque se trata de un proyecto con intensa fuerza, no está exento de copiosas controversias teóricas e ideológicas que, inexorablemente, irán en aumento. Aún no se atisba una solución integradora. Ésta parece hoy en día imposible, y quizás también se carezca de la voluntad de conseguirla.

El presente abordaje presenta algunas limitaciones. Entre ellas, y por razones de espacio, se ha reducido la bibliografía crítica con la PsP esgrimida en el trabajo. Otra limitación, aunque de signo contrario al anterior, que se dispone de una amplia cantidad de referencias, no expuestas aquí, de resultados positivos y prometedores acerca de la utilidad 
práctica de cierta PsP. Se impone, por tanto, la necesidad de procurar un punto de equilibrio acerca de los aspectos positivos, negativos y críticos de la PsP. En cualquier caso, parece que siempre consistirá en una solución parcial, pues alcanzar un acuerdo, actualmente, se vislumbra poco probable.

\section{Referencias}

Adler, A., \& Seligman, M. E. P. (2016). Using wellbeing for public policy: Theory, measurement, and recommendations. International Journal of Wellbeing, 6, 1-35. doi:10.5502/ijw.v6i1.429

Adorno, T. W. (1970/1980). Teoría estética. Madrid: Taurus.

American Psychiatric Association. (2014). Manual diagnóstico y estadístico de los trastornos mentales (DSM-V). Madrid, España: Panamericana.

American Psychological Association. (2010). Manual de publicaciones de la American Psychological Association. México, DF: Manual Moderno.

American Psychological Association. (2017). Ethical Principles of Psychologists and Code of Conduct (Effective June 1, 2003 (as amended 2010, 2016). Effective January 1, 2017). Washington, DC: American psychological Association.

Aristóteles (1985). Ética Nicomáquea. Madrid, España: Gredos.

Aristóteles (1994). Retórica. Madrid, España: Gredos.

Bal, M. (2002/2009). Conceptos viajeros en las humanidades: una guía de viaje. Murcia, España: CENDEAC.

Bandura, A. (2008). An agentic perspective on positive psychology. En S. J. Lopez (Ed.), Positive psychology (Vol. 1, pp. 167-196). Westport, CT: Greenwood Publishing Company.

Barlow, D. H. (2010). Negative effects from psychological treatments. A perspective. American Psychologist, 65, 13-20. doi: 10.1037/a0015643

Bok, D. (2010). The politics of happiness. Princeton, NJ: Princeton University Press.

Bolier, L., Haverman, M., Westerhof, G. J., Riper, H., Smit, F., \& Bohlmeijer, E. (2013). Positive psychology interventions: A meta-analysis of randomized controlled studies. BMC Public Health, 13, 119. doi: 10.1186/1471-2458-13-119

Braudel, F. (1958/2002). La larga duración. En F. Braudel (Ed.), Las ambiciones de la historia (pp. 147-177). Barcelona, España: Crítica.

Brown, N. J. L., Lomas, T., \& Eiroa-Orosa, F. J. (Eds) (2017). The Routledge international handbook of critical positive psychology. Oxford, UK: Routledge.

Bruckner, P. (2000). L'euphorie perpétuelle. Essai sur le devoir de bonheur. Paris: Grasset et Frasquelle.

Buela-Casal, G. (2002). La evaluación de la investigación científica: “el criterio de la mayoría": El factor de impacto, el factor de prestigio y "Los Diez Mandamientos para incrementar las citas". Análisis y Modificación de Conducta, 28, 455-476.

Buela-Casal, G. (2014). Pathological publishing: a new psychological disorder with legal consequences? The European Journal of Psychology Applied to Legal Context, 6, 91-97. doi: 10.1016/j.ejpal.2014.06.005

Caprara, G. V., Eisenberg, N., \& Alessandri, G (2017). Positivity: The dispositional basis of happiness. Journal of Happiness Studies, 18, 353-371. doi: 10.1007/s10902-016-9728-y

Carver, C. S., Scheier, M. F., y Segerstrom, S. C. (2010). Optimism. Clinical Psychology Review, 30, 879-889. doi: 10.1016/j.cpr.2010.01.006

Cederström, C., \& Spicer, A. (2015). The wellness syndrome. Cambridge, UK: Polity Press.

Cicerón, M. T. (2005). Disputaciones tusculanas. Madrid, España: Gredos. Cicerón, M. T. (2014). Sobre los deberes. Madrid, España: Gredos.

Coyne, J. C., Pajak, T. F., Harris, J., Konski, A., Movsas, B., Ang, K., $\&$ Watkins Bruner. D. (2007). Radiation therapy oncology group. Emotional well-being does not predict survival in head and neck cancer patients: a Radiation Therapy Oncology Group study. Cancer, 1, 2568-2575. doi: 10.1002/cncr.23080

Coyne, J. C., \& Tennen, H. (2010). Positive psychology in cancer care: Bad science, exaggerated claims, and unproven medicine. Annals of Behavioral Medicine, 39, 16-26. doi: 10.1007/s12160-009-9154-z

Coyne, J., C., Tennen, H., \& Ranchor, A. V. (2010). Positive psychology in cancer care: A story line resistant to evidence. Annals of Behavioral Medicine, 39, 35-42. doi: 10.1007/s12160-010-9157-9

Diener, E., Heintzelman, S. J., Kushlev, K., Tay, L., Wirtz, D., Lutes, L. D., \& Oishi, S. (2017). Findings all psychologists should know from the new science on subjective well being. Canadian Psychology, 58, 87-104. doi: 10.1037/cap0000063

Ehrenreich, B. (2009/2011). Sonríe o muere. Madrid: Turner.

Epicuro (1996). Obras completas. Madrid, España: Cátedra.

Espeland, W. N., \& Sauder, M. (2016). Engines of anxiety. Nueva York, NY: Russell Sage Foundation.

Ferguson, E. (2016). Empathy: "The good, the bad and the ugly". En A. Wood y J. Johnson (Eds.), Positive clinical psychology (pp. 103-125). Chichester, UK: Wiley y Sons.

Fernández-Ríos, L. (2008). Una revisión crítica de la psicología positiva. Revista Colombiana de Psicología, 17, 161-176.

Fernández-Ríos, L., \& Cornes, J. M. (2009). A critical review of the history and current status of positive psychology. Anuario de Psicología Clínica y de la Salud, 5, 7-13.

Fernández-Ríos, L., \& Novo, M. (2012). Positive Psychology: Zeigeist (or spirit of the times) or ignorance (or disinformation) of history? International Journal of Clinical and Health Psychology, 12, 333-344.

Fernández-Ríos, L., \& Rodríguez-Díaz, J. (2014). The "impact factor style of thinking": A new theoretical framework. International Journal of Clinical and Health Psychology, 14, 154-160. doi: 10.1016/S16972600(14)70049-3

Fernández-Ríos, L., \& Vilariño, M. (2016). Mitos de la psicología positiva: maniobras engañosas y pseudociencia. Papeles del Psicólogo, $37,134-142$.

Ford, B. Q., \& Mauss, I. B. (2014). The paradoxical effects of pursuing positive emotion: When and why wanting to feel happy backfires. En J. Gruber y J. T. Moskowitz (eds.), Positive emotion: Integrating the light sides and dark sides (p. 363-381). Oxford, NY: Oxford University Press.

Ford, B. Q., Mauss, I. B., \& Gruber, J. (2015). Valuing happiness is associated with bipolar disorder. Emotion, 15, 211-222. doi:10.1037/ emo0000048 
Ford, B. Q., Shallcross, A. J., Mauss, I. B., Floerke, V. A., \& Gruber, J. (2014). Desperately seeking happiness: Valuing happiness is associated with symptoms and diagnosis of depression. Journal of Social and Clinical Psychology, 33, 890-905. doi: 10.1521/jscp.2014.33.10.890

Forgas, J. P. (2013). The upside of feeling down: The benefits of negative mood for social cognition and behaviour. En J. P. Forgas, K. Fiedler y C. Sedikides (Eds.), Social thinking and interpersonal behavior (pp. 221-238). New York, NY: Psychology Press.

Forgas, J. (2017). Can sadness be good for you? On the cognitive, motivational, and interpersonal benefits of mild negative affect. Australian Psychologist, 52, 3-13. doi:10.1111/ap.12232

Frances, A. (2013/2014). ¿Somos todos enfermos mentales? Madrid, España: Ariel.

Gillham, J. E., \& Seligman, M. E. P. (1999). Footsteps on the road to positive psychology. Behaviour Research and Therapy, 37, S163-S173. doi: 10.1016/S0005-7967(99)00055-8

Gruber, J., Mauss, I. B., \& Tamir, M. (2011). A dark side of happiness? How, when, and why happiness is not always good. Perspectives on Psychological Science, 6, 222-233. doi: 10.1177/1745691611406927

Gruber, J., \& Moskowitz, J. T. (Eds.) (2014). Positive emotions. New York, NY: Oxford University Press.

Gruber, J., Van Meter, A., Gilbert, K. E., Youngstrom, E. A., Kogos-Youngstrom, J., Feeny, N. C., \& Findling, R. L. (2017). Positive emotion specificity and mood symptoms in an adolescent outpatient sample. Cognitive Therapy and Research, 41, 304-319. doi: 10.1007/ s10608-016-9796-7

Haslam, N. (2016). Concept creep: Psychology's expanding concepts of harm and pathology. Psychological Inquiry, 27, 1-17. doi: 10.1080/1047840X.2016.1082418

Held, B. S. (2002). The tyranny of the positive attitude in America: Observation and speculation. Journal of Clinical Psychology, 58, 965-992. doi: 10.1002/jclp.10093

Held, B. S. (2004). The negative side of positive psychology. Journal of Humanistic Psychology, 44, 9-46. doi: 10.1177/0022167803259645

Held, B. S. (2016). Why clinical psychology should not go "positive" -and/ or "negative". En A. Wood y J. Johnson (Eds.), The Wiley handbook of positive clinical psychology (pp. 31-46). Chichester, UK: Wiley y Sons.

Henrich, J., Heine, S. J., \& Norenzayan, A. (2010). The weirdest people in the world? Behavioral and Brain Sciences, 33, 61-83. doi: 10.1017/ S0140525X0999152X

Horwitz, A. V., \& Wakefield, J. C. (2007). The loss of sadness. Oxford, NY: Oxford University Press.

Ivtzan, I., \& Lomas, T. (2016). Mindfulness in positive psychology: The science of meditation and wellbeing. London, UK: Routledge.

Johnson, E. L. (2017). Towards a Christian positive psychology: Introduction to the special issue. The Journal of Positive Psychology, 12, 425-426. doi: 10.1080/17439760.2016.1228011

Kashdan, T. B., \& Biswas-Diener, R. (2014). The upside of your dark side. New York, NY: Hudson/Penguin.

Klimecki, O., \& Singer, T. (2012). Empathic distress fatigue rather than compassion fatigue? Integrating findings from empathy research in psychology and social neuroscience. En B. Oakley, A. Knafo, G. Madhavan y D. S. Wilson (Eds.), Pathological altruism (pp. 368-383).
New York, NY: Oxford University Press.

Koselleck, R. (2006/2012). Historia de los conceptos. Madrid: Trotta.

Kuhn, T. S. (1962/2006). Las estructura de las revoluciones científicas. México, D. F.: Fondo de Cultura Económica.

Larsen, T. J. (2017). Introduction to the Special Section on mixed emotions. Emotion Review, 9, 97-98. doi: 10.1177/1754073916672523

Lillo-Redonet, F. (2001). Palabras contra el dolor: la consolación filosófica latina de Cicerón a Frontón. Madrid, España: Ediciones Clásicas.

Liu, B., Floud, S., Pirie, K., \& for the Million Women Study Collaborators (2006). Does happiness itself directly affect mortality? The prospective UK million women study. The Lancet, 387, 874-881. doi: 10.1016/ S0140-6736(15)01087-9

Lomas, T. (2016). The positive power of negative emotions. London, UK: Piatkus.

Lyubomirsky, S., King, L. A., \& Diener, E. (2005). The benefits of frequent positive affect. Psychological Bulletin, 131, 803-855. doi: 10.1037/0033-2909.131.6.803

Marco Aurelio (1983). Meditaciones. Madrid, España: Gredos.

McDonald, M., \& Wearing, M. (2017). Positive psychology and its discontents. Oxford, UK: Routledge.

McGuirk, L., Kuppens, P., Kingston, R., y Bastian, B. (2017). Does a culture of happiness increase rumination over failure? Emotion. doi: 10.1037/emo0000322.

Norem, J. K. (2001). The positive power of negative thinking. New York, NY: Basic Books.

Oakley, B. A. (2013). Concepts and implications of altruism bias and pathological altruism. PNAS, 110, suppl. 2, 10408-10415. doi: 10.1073/ pnas. 1302547110

Oishi, S. (2012). The psychological wealth of nations. Malden, MA: Wiley-Blackwell.

Ovidio (2008). Tristes pónticas. Madrid, España: Gredos.

Park, N., Peterson, C., \& Sun, J.K. (2013). La Psicología Positiva: Investigación y aplicaciones Positive Psychology: Research and practice. Terapia Psicológica, 31, 11-19. doi: 10.4067/S0718-48082013000100002

Parrott, W.G. (Ed.) (2014). The positive side of negative emotions. New York, NY: Guilford Press.

Pawelski, J. (2016a). Defining the 'positive' in positive psychology: Part I. A descriptive analysis. The Journal of Positive Psychology, 11, 339-356. doi: 10.1080/17439760.2015.1137627

Pawelski, J. (2016b). Defining the 'positive' in positive psychology: Part II. A normative analysis. The Journal of Positive Psychology, 11, 357-365. doi: 10.1080/17439760.2015.1137628

Pérez-Álvarez, M. (2013). La psicología positiva y sus amigos: en evidencia. Papeles del Psicólogo, 34, 208-226.

Peterson, C., \& Seligman, M. E. P. (2004). Character strengths and virtues. Washington, DC: American Psychological Association.

Piña, J. A. (2014). La psicología positiva: ¿Ciencia y práctica de la psicología? Papeles del Psicólogo, 35, 144-158.

Platón (1986). La República. Madrid, España: Gredos.

Plutarco (1986). Escrito de consolación a Apolonio. En Plutarco, Obras morales y de costumbres (Moralia) (vol. 2) (pp. 7-113). Madrid, España: Gredos. 
Quoidbach, J., Mikolajczak, M., \& Gross, J. (2015). Positive interventions: An emotion regulation perspective. Psychological Bulletin, 141, 655693. doi: $10.1037 / \mathrm{a} 0038648$

Ryan, R. M., \& Deci, E. L. (2017). Self-determination theory. New York, NY: Guilford Press.

Santos-Rego, M. A., \& Fernández-Ríos, L. (2016). El factor de impacto y el futuro de las revistas académicas. El riesgo de patologización. Innovación Educativa, 16, 35-51

Scherer, K. R., \& Mehu, M. (Eds.) (2015). Normal and abnormal emotions - The quandary of diagnosing affective disorder: Introduction and overview. Emotion Review, 7, 201-203. doi: 10.1177/1754073915576689

Seligman, M. E. P. (1998). Building human strength: Psychology's forgotten mission. APA Monitor, 29.

Seligman, M. E. P. (1999). The president's address [printed in the 1998 APA annual report]. American Psychologist, 54, 559-562.

Seligman, M. E. P. (2008). Positive health. Applied Psychology: An International Review, 57, 3-18. doi: 10.1111/j.1464-0597.2008.00351.x

Seligman, M. E. P. (2011). La vida que florece. Barcelona, España: Ediciones B.

Seligman, M. E. P., y Csikszentmihalyi, M. (2000). Positive psychology: An introduction. American Psychologist, 55, 5-14. doi: 10.1037/0003066X.55.1.5

Séneca, L. A. (1996a). Consolación a Marcia. Madrid, España: Gredos.

Séneca, L. A. (1996b). Consolación a su madre Helvia. Madrid, España: Gredos.

Séneca, L. A. (1996c). Consolación a Polibo. Madrid, España: Gredos.

Sheldon, K. M. (2011). What's positive about positive psychology? Reducing value-bias and enhancing integration within the field. En K. M. Sheldon, T. Kashdan \& M. Steger (Eds.), Designing the future of positive psychology (pp. 421-429). Oxford, NY: Oxford University Press.

Sin, N. L., \& Lyubomirsky, S. (2009). Enhancing well-being and alleviating depressive symptoms with positive psychology interventions: a practice-friendly meta-analysis. Journal of Clinical Psychology, 65, 467-487. doi: 10.1002/jclp.20593

Snyder, C. R., \& Lopez, S. J. (2007). Positive psychology. Thousand Oaks, CA: Sage Publications.

Widiger, T. A. (2016). An integrative model of personality strengths and weaknesses. En A. M. Wood y J. Johnson (Eds), The Wiley handbook of positive clinical psychology (pp. 261-277). Chichester, UK: Wiley y Sons.

Wittgenstein, L. (1954/2017). Investigaciones filosóficas. Madrid, España: Trotta.

Wood, A. M., Emmons, R. A., Algoe, S. B., Froh, J. J., Lambert, N. M., \& Watkins, P. (2016). A dark side of gratitude? Distinguishing between beneficial gratitude and its harmful impostors for the positive clinical psychology of gratitude and well-being. En Wood, A. M. \& Johnson, J. (Eds), The Wiley handbook of positive clinical psychology (pp. 137-151). Chichester, UK: Wiley y Sons. 